\title{
Analysis of 5G-TSN Integration to Support Industry 4.0
}

\author{
Ana Larrañaga ${ }^{1}$, M. Carmen Lucas-Estañ ${ }^{2}$, Imanol Martinez ${ }^{1}$, Iñaki Val ${ }^{1}$, Javier Gozalvez ${ }^{2}$ \\ ${ }^{1} \mathrm{HW}$ and Communication Systems Area, Ikerlan Technology Research Centre, Mondragón 20500, Spain \\ ${ }^{2}$ UWICORE Laboratory, Universidad Miguel Hernández de Elche (UMH), Elche 03202, Spain \\ \{ana.larranaga, imartinez, IVal\}@ikerlan.es, \{m.lucas,j.gozalvez\}@umh.es
}

\begin{abstract}
Time Sensitive Networking (TSN) is becoming the standard Ethernet-based technology for converged networks of Industry 4.0 due to its capacity to support deterministic latency requirements. However, it cannot provide the required flexibility to support mobile industrial applications required for the factories of the future. This could be enabled through the integration of wireless technologies in factories, and in particular of $5 \mathrm{G}$ and Beyond networks since they have been designed to support ultrareliable and low-latency communications. This has triggered significant interest to integrate 5G and TSN networks, and first frameworks for such integration have been defined. However, the work is at early stages and the solutions to effectively integrate the two networks so that 5G can support TSN QoS levels are yet to be designed. This paper discusses current research and standardization work on 5G-TSN integration, and quantifies for a closed loop control application the 5GS bridge delay. The paper uses an example based on 5G-ACIA [1] to discuss open technical and research challenges to effectively integrate 5G and TSN.
\end{abstract}

Keywords-TSN; 5G; 5G-TSN; Deterministic; Time-critical; Industry 4.0; QoS support

\section{INTRODUCTION}

Future smart factories will provide wireless and wired connectivity between the entire production line in order to exchange data and take decisions autonomously. To achieve this paradigm, highly reliable and deterministic communications will be key. In the last decade, industry has introduced wired communications in order to support the strict requirements that it needs. Time-Sensitive Networking (TSN) Ethernet-based technology is being developed to provide deterministic, real-time and ultra-reliable communications. However, TSN cannot guarantee the flexibility required by the factories of the future to support mobility in some industrial applications. Wireless technologies can provide the required flexibility, and the integration of TSN with wireless technologies will be key. $5^{\text {th }}$ Generation (5G) networks are considered one of the best options to support the Industry 4.0 paradigm as it has been designed to support Ultra-Reliable Low Latency Communications (URLLC). This paper discusses the current procedures defined in 3GPP standards for $5 \mathrm{G}$ and TSN integration, and illustrates with a closed loop control application example based on 5G-ACIA [1] how to quantify the $5 \mathrm{GS}$ bridge delay as a relevant component for an effective $5 \mathrm{G}$ TSN integration. The example is used to identify existing open technical and research questions and limitations that require further study for an effective and efficient 5G-TSN integration.

The rest of the paper is structured as follows. Section II and III explain the most important TSN and 5G-NR (New Radio) features. Section IV introduces the state of the art of 5G-TSN integration. Section V presents the use case and analyzes the current procedures defined for $5 \mathrm{G}$ and TSN integration. Section VI presents main conclusions.

\section{Time SENSITIVE NETWORKING (TSN)}

TSN is an extension of traditional Ethernet data-link layer developed to support deterministic transmissions with real time and high reliability requirements. TSN is a set of layer 2 open standards specified by IEEE 802.1 .

A TSN network is composed of end stations that are the source and destination of the TSN streams (also referred to as talkers and listeners respectively), and bridges that are special Ethernet switches capable of transmitting and receiving Ethernet frames of a TSN stream following a schedule. TSN standard IEEE 802.1Qcc [2] defines three network management models: a fully distributed model, a centralized network and distributed user model, and a fully centralized one. This paper focuses on the fully centralized model since it is the one that allows the most efficient use of network resources. In this model, the TSN bridges communicate their network capabilities and active topology information to the CNC (Centralized Network Configuration) node. End stations send their TSN stream requirements to the CUC (Centralized User Configuration) node that forwards this information to the $\mathrm{CNC}$ (the CUC may adapt these TSN end station stream requirements before forwarding them to the $\mathrm{CNC}$ ); the $\mathrm{CNC}$ has then a complete view of the TSN network and it can determine the configuration of each bridge (e.g. the scheduling) to fulfil the TSN stream requirements. The CNC can send specific TSN configuration information to the CUC, which may send configuration information to the end stations.

TSN defines a Time Aware Shaper (TAS) scheduler in IEEE 802.1Qbv [2] for guaranteeing the transmission of high priority deterministic traffic in a bounded time. To this end, the Ethernet traffic is divided into different classes. There can be up to 8 traffic classes and Best Effort (BE) is the default traffic class. TAS scheduler establishes on every egress port of each bridge the time-interval each traffic class has access to the channel so that the time requirements of high priority traffic are met. TAS scheduler creates a time-protected channel used by TSN traffic class in order to guarantee their deterministic transmission time. When data enters in a TSN bridge, it is forwarded to the corresponding egress port. The data is classified in different queues based on the traffic class. Each queue has different priority, from less priority 0 to high priority 7. The queues are controlled by a Gate Control Lists (GCL) that determines which queues are opened or closed at each time 
instant. If there are more than one queue opened at the same time, the forwarding of frames depends on the priority of the queue. In order to support TAS scheduling, all the nodes (talkers, listener and bridges) in the network are synchronized using one master and several slaves' clocks, as defined in the IEEE 802.1AS standard [3].

\section{5G SYSTEM}

5G System (5GS) has two main network components: the Radio Access Network (RAN) and the Core Network (CN). The RAN includes the UE (User Equipment) and the gNB (new generation Node $\mathrm{B}$ ). The $5 \mathrm{G} \mathrm{CN}$ is highly flexible and consists of a series of independent network functions for the user plane (UP) and the control plane (CP) [4]. UP carriers the network user traffic and $\mathrm{CP}$ carries signaling and control traffic. The UPF (User Plane Function) handles the UP path between the gNB and the external data network (e.g. a TSN network). In the control plane, the SMF (Session Management Function) interacts with the user plane to establish or modify PDU (Protocol Data Unit) sessions that define the connectivity services between UE and the data network. The PCF (Policy Control Function) provides policy rules to the SMF. The AF (Application Function) interacts with the PCF for policy control and can influence on traffic routing in the UP. To support low latency communications, $5 \mathrm{G}$ reduces the processing times at $\mathrm{UE}$ and gNB, defines more flexible frames structures, and the use of shorter transmission slots based on different numerologies. These numerologies represent the spacing between sub-carriers and result in slots from 1 to $0.0625 \mathrm{~ms}$. It is possible to transmit only using part of a slot, referred to as minislot. Another key aspect to reduce latency is the definition of grant-free scheduling for uplink transmissions: gNB preallocates resources for a UE. When the UE wants to transmit, it does not need to ask resources to the gNB (skipping the delay associated to this process), and it can transmit data immediately.

5G supports different QoS Flows depending on the service (a QoS Flow is the finest granularity of QoS differentiation in a PDU Session). A QoS Flow in 5GS may either be GBR (Guaranteed Bit Rate) or Non-GBR. GBR QoS Flows may use either GBR or Delay-critical GBR resources. Each QoS Flow is related with a $5 \mathrm{G}$ QoS profile that contains, among others, the following QoS parameters: the guaranteed and maximum flow bit rate (GFBR and MFBR respectively), maximum packet loss rate, and a $5 \mathrm{G}$ QoS Identifier (5QI). The 5QI is a scalar that is used as a reference to a set of QoS characteristics: priority, packet delay budget (PDB), packet error rate (PER), and maximum data burst volume (MDBV). The PDB is the maximum time that a packet may be delayed between the UE and the UPF that connects to the data network. The PDB is explicitly divided into RAN PDB and CN PDB. The MDBV is the largest amount of data that the RAN is required to serve within a period of RAN PDB. 5QI values can be standardized in [4], preconfigured in the RAN or dynamically defined.

\section{INTEGRATION OF 5G AND TSN}

This section presents some of the recent proposals for integration of TSN and 5G that are currently being studied by 3GPP, Avnu Alliance and 5G-ACIA, among others.

\section{A. Architecture for the 5G-TSN Integration}

3GPP Release 16 [4] defines the basic functionalities and architecture to support Time Sensitive Communications (TSC) and integrate TSN and 5G. 3GPP Release 16 focused first on TSN networks with fully centralized management. 5GS is integrated within the TSN network as a logical TSN bridge. 3GPP introduces a TSN translator (TT) functionality at the interconnection points between both networks, that is in the device side (DS) and in the network side (NW). The DS-TT and NW-TT act as TSN ingress and egress Ethernet ports in the logical 5GS TSN bridge. Thanks to the TT functionality, the 5GS appears as any other TSN bridge for the TSN network. The DS-TT and NW-TT are connected to UE and UPF respectively, and their functionality is to configure all parameters necessary to coordinate $5 \mathrm{G}$ and TSN. In the control plane, the TSN AF is in charge of exchanging control data between 5GS and the TSN $\mathrm{CNC}$ in order to configure $5 \mathrm{GS}$ as a TSN bridge.

\section{B. $5 G S$ bridge information}

5GS reports 5GS bridge information to the TSN network. This information is used by the CNC to adequately configure the TSN network so that it supports the QoS requirements of TSN traffic. The 5GS bridge information includes information about bridge ID, ethernet ports and their MAC address, neighbor discovery information, supported minimum/maximum bridge delay, propagation delay and traffic classes. The 5GS calculates the bridge delay for each port pair and traffic class. The bridge delay represents the time that a frame for a specific traffic class takes to be forwarded from an ingress port to an egress port. The bridge delay (BD) is calculated as follows:

$$
B D=t_{\text {residence,UE-DSTT }}+d_{\text {dependent }}+d_{\text {independent }}
$$

where $t_{\text {residence,UE-DSTT }}$ is the time that a packet needs to be forwarded from DS-TT to UE and vice versa and is referred to as residence time, $d_{\text {dependent }}$ is the delay experienced in the bridge that is dependent on the frame length, and $d_{\text {independent }}$ is the delay to store and forward a frame through the bridge and that is independent of the frame length; $d_{\text {independent }}$ does not depend on the frame length but is a function of the processing time, the 5G frame structure, scheduling, HARQ, etc [5].

\section{C. $5 G S$ bridge configuration}

5GS needs information about the QoS requirement of each TSN stream to adequately configure the different management mechanisms at the RAN and CN. QoS requirements of a TSN stream are identified by a stream ID, a priority, a traffic class, a maximum frame size, a maximum latency and source/destination of the flow, among other parameters. In order to support TSN streams in 5G, the TSN QoS requirements need to be mapped to a $5 \mathrm{G}$ QoS profile. TSN streams are supported in 5GS as Delay-critical GBR QoS flows. The 5QI selected for the TSN QoS flow should have a MDBV equal to or higher than the TSC Burst Size, and a PDB that is equal or higher than the bridge delay for this traffic class. The QoS mapping between TSN QoS traffic class and 5G QoS profiles is carried out at the TSN AF and the PCF. The TSN AF decides the TSN QoS parameters (i.e. priority and delay) based on the received configuration information from the CNC. The TSN QoS information is sent to the PCF, which is a function of the 
5G CN that maps the TSN QoS parameters into a 5QI. 3GPP standards also define the exchange of additional TSC Assistance Information (TSCAI) that may be used in the 5GS for a more efficient scheduling of periodic and deterministic traffic flows in the RAN. TSCAI provides traffic pattern parameters such as burst arrival time with reference to the ingress port, periodicity, and flow direction.

\section{BRIDGE DELAY ESTIMATION FOR AN EFFECTIVE 5G-TSN INTEGRATION}

Our research focuses on how to effectively integrate $5 \mathrm{G}$ and TSN to support latency-critical and deterministic industrial applications. The 5GS bridge and TT functionalities have a strong impact on this integration, so in this first study, we analyze the bridge delay considering the procedures used to establish an integrated 5G-TSN connection with TSN traffic. For the analysis, we consider an industrial scenario based on 5G-ACIA white paper [1] where the 5G-TSN network supports the connectivity of a logistic closed loop control use case. For this use case, a 5G UE is locally connected (wired) to one sensor and one actuator that communicate through the $5 \mathrm{G}$ wireless connection with a remote PLC (Programable Logic Controller). The sensor sends information to the PLC every $1 \mathrm{~ms}$. In the next control cycle, the PLC sends a command to the actuator. In general, during the same control cycle the sensor writes data in the PLC and the actuator reads the data from the PLC. However, in this example the actuator reads the data transmitted from the PLC one control cycle later. Figure 1 shows the analyzed 5GTSN architecture, that considers the deployment of a private $5 \mathrm{G}$ network with a dedicated local core network. The PLC is connected to a TSN bridge by means of a cable. The scenario includes two office computers that exchange plant information, but their data is considered best effort traffic. We focus the rest of the analysis on higher priority TSN traffic.

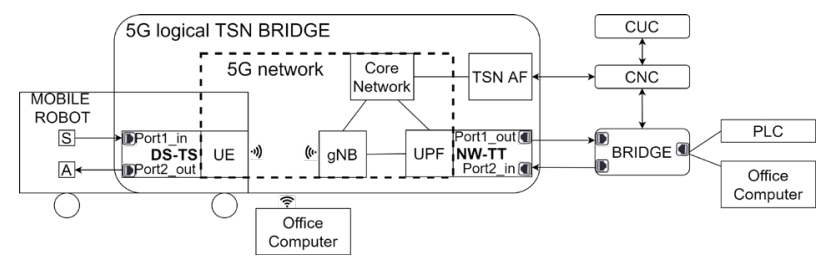

Figure 1. Example of 5G-TSN integrated network architecture.

Initialization: Talkers and listeners send requirements information to the CUC (Table 1) that forwards this information to the CNC. We assume only two streams of TSN traffic class.

TABLE I. TALKER REQUIREMENTS

\begin{tabular}{|c|c|c|}
\hline Talker & Sensor (S) & PLC \\
\hline Stream ID & Stream 1 & Stream 2 \\
\hline Priority & 4-7 (TSN traffic, highest priority) \\
\hline MaxFrameSize & 64 Bytes & 256 Bytes \\
\hline FramePerInterv. & \multicolumn{2}{|c|}{1} \\
\hline Interval (Periodicity) & \multicolumn{2}{|c|}{$1 \mathrm{~ms}$} \\
\hline Maximum Latency & \multicolumn{2}{|c|}{$1 \mathrm{~ms}$} \\
\hline
\end{tabular}

Physical network topology and bridge capabilities: The $\mathrm{CNC}$ has to discover the physical network topology and the TSN capabilities of each bridge. To this end, each bridge sends to the CNC the following data: bridge ID, ethernet ports and their MAC address, neighbor discovery information, supported minimum/maximum bridge delay, propagation delay and traffic classes. Equation (1) and Table 1 parameters are used in order to calculate the minimum and maximum bride delay of the TSN and 5GS bridge. Minimum bridge delays are calculated using the stream 1 (from sensor to PLC) which represents the minimum streams size, while maximum bridge delays are calculated using the stream 2 (from PLC to actuator) that represents the maximum stream size.

We consider that, when a TSN stream arrives to the egress port of the TSN bridge, it is transmitted immediately (we consider that the gate is open for this traffic class). In this case, the delay experienced in the bridge includes processing and transmission times. We consider a processing delay in the TSN bridge equal to $1.5 \mu$ s as presented in [6]. If we assume the use of a link with data bit rate equal to $100 \mathrm{Mbps}$ (typical bit rate in industry for latencies between $25 \mu \mathrm{s}-2 \mathrm{~ms}$ [7]), the minimum and maximum dependent delay (equal to the transmission time) can be computed as:

$$
\begin{aligned}
& d_{\text {dependent }, \text { TSNbridge }}^{\min }=\frac{64 \text { Bytes } \times 8 \text { bits }}{100 \mathrm{Mbps}}=5.12 \mu \mathrm{s} \\
& d_{\text {dependent }_{\text {TSNbridge }}}^{\max }=\frac{256 \text { Bytes } \times 8 \text { bits }}{100 \mathrm{Mbps}}=20.5 \mu \mathrm{s}
\end{aligned}
$$

Finally, the minimum and maximum TSN bridge delays $\left(B D_{\text {TSNbridge }}^{\min } B D_{\text {TSNbridge }}^{\max }\right)$ are given by:

$$
\begin{aligned}
& B D_{\text {TSNbridge }}^{\min }=5.12 \mu \mathrm{s}+1.5 \mu \mathrm{s}=6.62 \mu \mathrm{s} \\
& B D_{\text {TSNbridge }}^{\max }=20.5 \mu \mathrm{s}+1.5 \mu \mathrm{s}=22 \mu \mathrm{s}
\end{aligned}
$$

In the $5 \mathrm{GS}$ bridge, we consider the use of a preconfigured $5 \mathrm{QI}$ in the RAN to calculate the bridge delay; this 5QI is associated to preconfigured values for the QoS parameters, PDB, MDBV and GFBR. These values are not standardized and can be established according to the network capabilities. In this section, we estimate the values for PDB, GFBR and MDBV that should be supported in this example. 5GS should support to transmit TSN stream 1 and stream 2 inside the required maximum latency. The PDB should be equal to or higher than the 5GS bridge delay. The PDB is calculated as:

$$
P D B \leq l_{\text {e2e }}-l_{\text {app }}-l_{\text {links }}-B D_{\text {TSNbridge }}
$$

where $l_{e 2 e}$ is the required end-to-end latency ( $\left.1 \mathrm{~ms}\right), l_{a p p}$ is the processing time at the application level in the end stations (we assume $l_{a p p}$ equal to the $25 \%$ of the end-to-end latency, i.e., $250 \mu \mathrm{s}), l_{\text {links }}$ represents the propagation time in the wired links ( $l_{\text {links }}$ is considered equal to 0 based on the short distances between devices), and $B D_{\text {TSNbridge }}$ is the TSN bridge delay as calculated in (4) and (5) for stream 1 and 2 respectively. Based on (6), PDB is equal to $743.38 \mu$ s and $728 \mu$ s for stream 1 and 2 respectively.

Using the estimated value for PDB, it is possible to calculate the minimum bit rate needs to be guaranteed as:

$$
\begin{aligned}
& \mathrm{GFBR}_{\text {stream } 1}>\frac{64 \text { Bytes } \times 8 \mathrm{bits}}{\mathrm{PDB}_{\text {stream } 1}}=688.7 \mathrm{kbit} / \mathrm{s} \\
& \mathrm{GFBR}_{\text {stream } 2}>\frac{256 \text { Bytes } \times 8 \mathrm{bits}}{\mathrm{PDB}_{\text {stream } 2}}=2813.1 \mathrm{kbit} / \mathrm{s}
\end{aligned}
$$

where $\mathrm{GFBR}_{\text {stream } 1}$ and $\mathrm{GFBR}_{\text {stream2 }}$ are the minimum bitrates to transmit stream 1 and stream 2 respectively. We compute the minimum MDBV that 5GS has to support to 
transmit stream 1 and stream 2, considering the use of Robust Header Compression (RHC) and 5G L2 header of 6 bytes [8]. RHC compresses the Ethernet header from 18 Bytes to 2 Bytes. In this case, the $M D B V_{\text {stream } 1}$ is equal to 54 Bytes and $M D B V_{\text {stream } 2}$ is equal to 246 Bytes. We can then estimate the maximum (stream 2) and minimum (stream 1) 5GS bridge delays as:

$$
\begin{aligned}
& B D_{5 G S}^{\min }<\frac{M D B V_{\text {stream } 1} \times 8}{\mathrm{GFBR}_{\text {stream } 1}} \approx 627 \mu \mathrm{S} \\
& B D_{5 G S}^{\max }<\frac{M D B V_{\text {stream } 2} \times 8}{\mathrm{GFBR}_{\text {stream } 2}} \approx 699.6 \mu \mathrm{S}
\end{aligned}
$$

After establishing a PDU session, TSN AF receives UE-DSTT residence time value and it deduces the in/out port pairs. So, the bridge delay for each port pair and traffic class is updated adding the $t_{\text {residence,UE-DSTT }}$. Finally, $B D_{5 G S}$ for the $5 \mathrm{GS}$ bridge is given by $\left(627 \mu s+t_{\text {residence,UE-DSTT }}\right)$ and $\left(699.6 \mu s+t_{\text {residence,UE-DSTT }}\right)$ and they have to be smaller or equal to the estimated PDBs, $743.38 \mu \mathrm{s}$ and $728 \mu$ s respectively.

Network configuration: When the $\mathrm{CNC}$ receives the bridges information, it calculates the gate control list or GCL of each egress port as well as the paths by which the streams have to be forwarded. In this use case there are two streams with different flow directions. The streams are transmitted through different egress ports within the same bridge, so each egress port has its on GCL. Figure 2 represents the communication path for stream1, as well as the time instant at which stream 1 arrives and leaves the 5GS bridge.

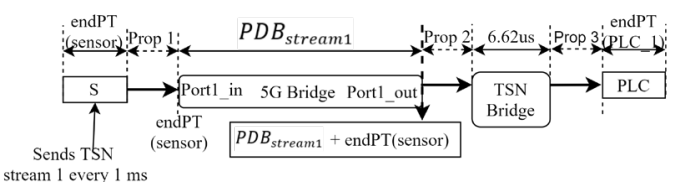

Figure 2. The end-to-end path of stream 1.

The CNC sends to the 5GS bridge information about the arrival time of each data stream and their periodicity. With this information, $5 \mathrm{G}$ can determine the traffic pattern information, make the QoS mapping, and configure the schedule. As presented in [9], the mapping between TSN stream priorities and 5QI priorities is flexible. 5G establishes priority values for standardized 5QI up to 90. Priority assigned for standardized 5 QI with delay critical GBR resources is lower than 24, and for the 5QI related to discrete automation applications is equal to 19. In this context, we consider a priority equal to or lower than 19. The QoS parameters for each QoS flow are presented in Table 2. The QoS profile and TSCAI information are sent to gNB in order to calculate the scheduling.

TABLE II. QOS PARAMETERS FOR THE DATA STREAMS

\begin{tabular}{|l|l|l|l|l|l|}
\hline & $\begin{array}{l}\text { Resource } \\
\text { type }\end{array}$ & Priority & MDBV & GFBR & PDB \\
\hline $\begin{array}{l}\text { QoS } \\
\text { UL }\end{array}$ & DelayCritical & $<19$ & $\begin{array}{l}64 \\
\text { Bytes }\end{array}$ & $>688.7 \mathrm{kbit} / \mathrm{s}$ & $\leq 743.4 \mu \mathrm{s}$ \\
\hline $\begin{array}{l}\text { QoS } \\
\text { DL }\end{array}$ & DelayCritical & $<19$ & $\begin{array}{l}256 \\
\text { Bytes }\end{array}$ & $>2813.1 \mathrm{kbit} / \mathrm{s}$ & $\leq 728 \mu \mathrm{s}$ \\
\hline
\end{tabular}

\section{CONCLUSIONS}

This WIP paper has discussed and analyzed the integration of $5 \mathrm{G}$ and TSN to support latency-critical and deterministic industrial applications. An important aspect of this integration is the bridge between the technologies. The paper quantifies the 5GS bridge delay for a close loop control industrial application example based on 5G-ACIA white paper [1]. From the work carried out in this paper, it is possible to identify some limitations that need to be further studied to effectively integrate $5 \mathrm{G}$ and TSN. For example, all standardized 5QIs in 5G (presented in [4]) establish a PDB equal to or higher than 5 ms. This PDB is not adequate for this kind of applications. Private 5G networks allow to deploy local $\mathrm{CN}$ that will allow to reduce considerably the delay experienced by data in the $\mathrm{CN}$. For example, Nokia presented in [9] that a CN PDB lower than $10 \mu$ s can be achieved considering a local optimized $\mathrm{CN}$ deployment. An adequate designed and dimensioning of the RAN of the 5GS will allow to meet PDB values lower than 1 $\mathrm{ms}$ as shown in [10]. Although pre-configured or dynamic 5QI values can be used, it might be interesting the definition of new standardized 5QI values for this kind of network deployments.

To support this kind of periodic traffic, the use of a semipersistent scheduling in DL or configured scheduling with periodic resources seems to be the most adequate scheduling options. However, it is important to analyze the case when the traffic periodicity does not match with the periodicities that can be supported in 5G RAN. How to address this issue needs to be further studied.

Finally, the 3GPP standard defines the basics concepts to estimate the 5GS bridge delay. However, the exact mapping between the 3GPP parameters and the bridge delay is not presented and is up to the implementation. How to estimate the bridge delay before initiating or establishing a QoS flow is a difficult task in 5GS. To this end, maybe it might be necessary to transmit some initial data to measure the PDB in 5GS before the TSN streams are established.

\section{REFERENCES}

[1] 5G-ACIA, "A 5G Traffic Model for Industrial Use Cases (White Paper)," no. November, 2019.

[2] IEEE, IEEE 802.1Q: Standard for Local and Metropolitan Area Networks--Bridges and Bridged Networks. 2018.

[3] IEEE, IEEE 802.1AS: Standard for Local and metropolitan area networks--Time and Synchronization for Time-Sensitive Applications in Bridged Local Area Networks. 2011

[4] 3GPP; Technical Specification Group Services and System Aspects; System architecture for the 5G System (5GS) Stage 2 (Release 16) 3GPP TS 23.501 V16.4.0, March, 2020.

[5] C. Mannweiler et al., "Reliable and deterministic mobile communications for industry 4.0" 24. ITG-Symposium Mob. Commun. - Technol. Appl., pp. 64-69, 2020.

[6] D. Pannell, "Choosing the Right TSN Tools to meet a Bounded Latency" in IEEE SA Ethernet \& IP @ Automotive Technology Day, September, 2019.

[7] A. Nasrallah et al., "Ultra-low latency (ULL) networks: The IEEE TSN and IETF DetNet standards and related 5G Ull research," IEEE Commun. Surv. Tutorials, vol. 21, no. 1, pp. 88-145, 2019.

[8] M. Khoshnevisan, "5G Industrial Networks With CoMP for URLLC and Time Sensitive Network Architecture," IEEE J. Sel. Areas Commun., vol. 37, no. 4, pp. 947-959, 2019.

[9] Nokia, "5G plug-and-produce. How the 3GPP 5G System facilitates Industrial Ethernet deployments (White Paper)." 2020.

[10] 3GPP; Technical Specification Group Radio Access Network; Study on self evaluation towards IMT-2020 Submission (Release 16) v16.1.0, September. 2019. 